\title{
La línea de pobreza subjetiva para Tunja, Colombia 2015
}

\author{
Subjective Poverty Line for Tunja, \\ Colombia 2015
}

A linha de pobreza subjetiva de Tunja -
Colombia 2015

Eliana Marcela Tobasura Jiménez * Julián Augusto Casas Herrera **

DOI: https://doi.org/10.19053/01203053.v36.n64.2017.6548

Fecha de recepción: 9 de septiembre de 2016

Fecha de aceptación: 25 de mayo de 2017

\footnotetext{
* Economista de la Universidad Pedagógica y Tecnológica de Colombia (UPTC). Tunja, Colombia. Correo: eliana. tobasura@gmail.com. (D) http://orcid.org/0000-0001-7011-9662

** Magister en Ciencias Económicas. Docente de la Escuela de Economía de la Universidad Pedagógica y Tecnológica de Colombia (UPTC). Tunja, Colombia. Correo: julian.casas01@uptc.edu.co (D) http://orcid.org/0000-00016606-5059 Dirección de correspondencia: calle 33 A\# 17 A 59. Tunja, Colombia.
} 


\section{Resumen}

La mayoría de los gobiernos del territorio nacional vienen anunciando, en los últimos años, descensos significativos de la pobreza como un éxito derivado de sus propios actos. Sin embargo, la percepción de los colombianos no concuerda con dichos descensos. Por ello, es pertinente establecer medidas complementarias, que tengan en cuenta la percepción de quienes se consideran pobres, con el propósito de erigir políticas públicas más certeras para mitigar y superar las consecuencias negativas de la pobreza. Este documento muestra una estimación de la línea de pobreza subjetiva, a partir de tres métodos, para la ciudad de Tunja en 2015, que permite evidenciar que el DANE subestimó la pobreza de la ciudad al menos en un $30 \%$.

Palabras clave: condiciones de vida, línea de pobreza subjetiva, medición y análisis de la pobreza.

Clasificación JEL: C01, C10, I30, I32. 
Apuntes CENES Volumen 36, Número 64

julio - diciembre 2017. Págs. 253-282

\section{Abstract}

Most governments of the departments of Colombia have been announcing significant decreases in poverty in recent years as a success resulted from their own actions. However, the perception of Colombians disagrees with these opinions. Therefore, it is important to establish complementary measures that take into account the perception of those who consider themselves to be in poverty, with the purpose of creating more accurate public policies to mitigate and overcome the negative consequences of poverty. This paper shows an estimate of the Subjective Poverty Line using three methods for Tunja in 2015, which indicates that DANE (Office of National Statistics of Colombia) has underestimated the poverty line in the city by at least $30 \%$.

Keywords: living conditions, subjective poverty line, measurement and analysis of poverty 


\section{Resumo}

A maioria dos governos dos departamentos da Colômbia tem anunciado reduções significativas da pobreza nos últimos anos, pois o sucesso resultou de suas próprias ações. No entanto, a percepção dos colombianos discorda dessas opiniões. Portanto, é importante estabelecer medidas complementares que levem em conta a percepção daqueles que se consideram na pobre$\mathrm{za}$, com o objetivo de criar políticas públicas mais precisas para mitigar e superar as conseqüências negativas da pobreza. Este artigo mostra uma estimativa da Linha de Pobreza Subjetiva usando três métodos para Tunja em 2015, o que indica que o DANE (Escritório de Estatísticas Nacionais da Colômbia) subestimou a linha de pobreza na cidade em pelo menos $30 \%$.

Palavras-chave: condições de vida, linha de pobreza subjetiva, medição e análise da pobreza 
Apuntes CENES Volumen 36, Número 64

julio - diciembre 2017. Págs. 253-282

\section{INTRODUCCIÓN}

La motivación de este documento se debe a que en los años recientes tanto el Gobierno nacional como los entes territoriales vienen anunciando descensos significativos en las tasas de pobreza. Sin embargo, la impresión que tiene la mayoría de la sociedad es que las cifras no concuerdan con lo que se percibe en la realidad. De hecho, las estrategias de protección social en Colombia se han incrementado en las últimas décadas y han puesto especial énfasis en estrategias de superación de la pobreza. Así pues, la pobreza en el ámbito nacional se redujo en 9.4 puntos porcentuales de 2010 a 2015.

No obstante, la percepción que tiene la sociedad sobre las condiciones en las que vive en la actualidad no encaja con las mejoras que presentan los indicadores de medición de pobreza objetiva. Por esta razón, este documento estima una línea de pobreza subjetiva (LPS) urbana para la ciudad de Tunja para 2015, a partir de tres métodos, con el propósito de contrastar los resultados oficiales frente a los que surgen desde la propia percepción de quienes sienten que viven en la pobreza.

Se destaca la importancia de este documento en cuanto trata el flagelo de la pobreza desde una visión subjetiva, en la que es el propio individuo quien evalúa su condición de vida respecto de su situación actual y el entorno que lo rodea, convirtiéndola en una medición complementaria a la medición objetiva de pobreza oficial. Lo que permitirá identificar elementos adicionales en el análisis de pobreza para Tunja y 
tener una comprensión integral sobre este fenómeno que permita brindar a los hacedores de políticas públicas, herramientas que contribuyan a destinar el gasto público social de manera eficiente, mediante el conocimiento de primera mano de las necesidades de los tunjanos $\mathrm{y}$, de este modo, ayuden a mejorar el bienestar de los mismos.

El documento consta de seis secciones. La primera es esta introducción. En la segunda se hace una breve revisión de los aspectos fundamentales de los enfoques de pobreza objetiva y subjetiva. En la tercera se muestra un análisis descriptivo del estado actual de la pobreza objetiva monetaria en Boyacá, Tunja y en el ámbito nacional. En la cuarta se presentan los detalles metodológicos que se tuvieron en cuenta para calcular la LPS de Tunja para el año 2015, a partir de tres métodos. En la quinta se analizan los resultados obtenidos. Finalmente, se hacen unos comentarios que recogen los principales aspectos tratados en el documento.

\section{POBREZA OBJETIVA Y SUBJETIVA}

La pobreza es un fenómeno que ha estado presente a lo largo de la historia del mundo. Con el paso de los años, los gobiernos han mostrado interés en superar este flagelo y, para ello, han intentado mejorar las condiciones económicas y sociales de los países a través de la implementación de políticas públicas, con el propósito de incrementar los niveles de vida y bienestar de sus habitantes.

Ante ello, la economía, como ciencia social, ha buscado establecer una explicación de la pobreza, desde su conceptualización hasta su forma de medición, a fin de identificar, localizar y cuantificar a los pobres existentes en una sociedad, así como distinguir cuáles son las carencias dentro del territorio que habitan, para poder plantear estrategias puntuales que aminoren los efectos de la pobreza y logren mejores niveles de vida, mediante el acceso de la población a un mayor número de bienes y servicios, a través de una mejor asignación y distribución de los recursos escasos.

Por ello, la pobreza se ha convertido en una preocupación esencial para la teoría económica, por tratarse de un factor determinante en el proceso de desarrollo de cualquier país. Sin embargo, al ser un fenómeno tan complejo no se ha podido llegar a un consenso sobre una definición y menos aún a una forma de medición única. En efecto, la conceptualización y comprensión de la pobreza se ha dificultado, porque se suele priorizar un aspecto, dejando de lado otros.

No obstante, su análisis se puede abordar desde lo general a lo específico. Desde la perspectiva general, la mayo- 
ría de autores concuerda en señalar que la pobreza es carencia o insuficiencia de un atributo (monetario o no monetario) con relación a un umbral por debajo del cual se hallará en pobreza (Casas \& Barichello, 2015a). Desde lo específico, el análisis de la pobreza se ha hecho desde cinco componentes: enfoques, definiciones, determinantes, consecuencias y tipos, y medición.

La explicación detallada de cada uno de los componentes escapa de los alcances de este documento (Casas \& Barichello, 2015a), dado que el interés es confrontar los resultados obtenidos por la línea de pobreza objetiva (en adelante LP) frente a la LPS. Para ello es preciso señalar que los dos métodos más utilizados en el mundo para medir la pobreza, son: necesidades básicas insatisfechas (NBI) y LP. El método de NBI opta por cinco atributos de bienestar y evalúa directamente y efectivamente si los hogares sufren cinco tipos de carencias (Casas, 2015a). Así, los integrantes de un hogar serán pobres si tienen al menos una NBI; pero si tienen más de una estarán en situación de miseria.

En cambio, el método de la LP opta por un único atributo de bienestar (la renta) con el fin de calcular los recursos que requiere un hogar/individuo para compararlo con el umbral $y$, de este modo, establecer si tiene o no la posibilidad de satisfacer sus necesidades básicas. De este método hay tres aproximaciones: LP relativa, LP absoluta y LPS. Estas dos últimas son objeto de atención en este documento. A continuación se exponen sucintamente sus principales aspectos metodológicos necesarios para su construcción.

\section{LP absoluta}

La estimación de esta se compone de dos líneas: LP alimentaria y LP no alimentaria. La metodología para el cálculo de la LP alimentaria establece dos pasos. El primero determina el requisito calórico mínimo necesario para mantener vivo a un individuo en reposo durante un día. El segundo establece el contenido y costo de una canasta de alimentos. El contenido de la canasta se desprende del cumplimiento de unos criterios que define el que la construye, tales como frecuencia de consumo de los alimentos. El costo de la canasta se suele determinar a partir de la utilización de los precios implícitos ${ }^{1}$ que arroja una Encuesta de Ingresos y Gastos para una población objetivo seleccionada.

Para el cálculo de la LP no alimentaria se organizan los hogares, de menor a mayor, de acuerdo con el valor gastado en los bienes, con el fin de seleccionar un grupo poblacional

${ }_{1}^{1}$ Salen de dividir el gasto del alimento entre la cantidad adquirida del mismo. 
objetivo, conocido como población de referencia. Esta sirve para definir la composición de la canasta y calcular el coeficiente de Orshansky $(\mathrm{CO})^{2}$. El valor del CO contempla los rubros de vivienda, transporte y vestuario. Una vez se tiene el valor del $\mathrm{CO}$, se multiplica por el valor de la LP alimentaria y se obtiene la LP (Instituo Nacional de Estadística de Uruguay -INE-, s.f.).

\section{LP subjetiva}

El análisis de la pobreza subjetiva intenta capturar la percepción que tienen las personas sobre su bienestar. Sin embargo, no ha sido muy usada en los países ni estandarizada por una institución como una metodología para la medición de la pobreza. Este enfoque se distingue de los enfoques de pobreza absoluta y relativa, pues estos, según Hagenaars y Van Praag (citados por Feres \& Mancero, 2001a) restringen el problema al criterio del investigador, quien define el concepto de pobreza y lo mide respecto de un umbral que él mismo ha establecido bajo ciertos parámetros considerados como mínimos, por lo que este método se encuentra libre de presunciones preestablecidas con anterioridad por el investigador.

Esta medición se aleja de las medidas objetivas, porque es manejado desde una perspectiva de abajo hacia arriba (bottom up). Según García (2002), en este análisis es el propio individuo quien valora su condición de vida y evalúa si el ingreso que posee es suficiente para suplir las necesidades de su familia para no considerarse pobre. Confrontar el valor de las cifras oficiales de pobreza con la posición del ciudadano sobre la percepción que tiene sobre su condición actual, nivel de vida y bienestar es de interés, debido a que sobre las personas que son consideradas como pobres a través de indicadores objetivos de pobreza monetaria, se ciernen los objetivos de política pública encaminados a su superación y además son los directamente afectados por esta condición.

En ese sentido, vale la pena destacar el debate que ha girado en torno de la aplicación del método de estimación de la LPS. En principio, se podría enunciar que hasta el momento ningún país ha adoptado el método de la LPS como metodología oficial para la estimación de las líneas de pobreza, porque hay riesgos en la implementación de políticas basadas en la LPS, tales como las dificultades que se presentarían a la hora de realizar comparaciones de las tasas de pobreza, tanto en el nivel internacional como nacional e incluso regional, pues hay un hecho que es irrefutable y es necesario enfatizar-

${ }^{2}$ Se obtiene de dividir los gastos totales entre el gasto de alimentos. 
lo: las personas inherentemente van a considerar sus recursos insu icientes (Colasanto, Kapteyn \& Van der Gaag, 1983). De ahí que las respuestas al cuestionamiento sobre el ingreso mínimo necesario tienden a inflar el dato suministrado, lo cual podría conducir a una sobreestimación de la verdadera pobreza vivida en una sociedad.

Sin embargo, el método de estimación de la LP absoluta o monetaria (léase objetiva) también contiene objeciones (Casas \& Barichello, 2015a). Por ejemplo, para la selección de la población de referencia hay un grado de subjetividad por parte del diseñador y encargado de llevar a cabo la medición de la pobreza. Para contextualizar este punto se debe indicar que el encargado de la medición tiene un propósito: estimar un porcentaje de pobreza para una sociedad. Por tanto, para hacerlo debe organizar todos los hogares, de menor a mayor, de acuerdo con el valor gastado en los bienes. Esto con el fin de seleccionar hogares que, por un lado, satisfagan sus necesidades alimentarias (para definir la composición de la canasta básica de alimentos), y por otro, que los hábitos de consumo en otras necesidades no alimentarias no resulten de decisiones sujetas a un marco de extrema escasez ni de gran abundancia económica (para establecer coeficiente de Orshansky). En Colombia, en las mediciones de pobreza se han elegido de tres tipos de pobla- ción de referencia ubicadas entre los percentiles 1 al 25, 30 al 59 y 1 al 90 . Pero nuevamente surge un cuestionamiento, ¿cuál es la correcta?

Posiblemente no haya una respuesta. De ahí que a la hora de hacer mediciones de pobreza exista un grado de arbitrariedad (léase subjetividad), pero esto no necesariamente invalida los resultados, puesto que después de todo estos se sustentan en la consistencia de los supuestos que adopta el encargado de la medición. En consecuencia, la discusión no debería ser sobre la subjetividad de un método o no, sino sobre su grado de explicación de la realidad, ya que todo ejercicio de medición debe aproximarse a explicarla lo más fielmente posible. Por tanto, esa es la valía que tiene el método de la LPS. Es decir, intenta capturar la realidad de sus dolientes. En ese sentido, el ejercicio de este documento va en esa dirección, debido a que en la sociedad colombiana, en general, existe una sensación de inconformismo sobre los porcentajes estimados de las tasas de pobreza por el Departamento Administrativo Nacional de Estadística (DANE) para el país, los departamentos y las principales ciudades. Por ello, a continuación se muestra el comportamiento de la pobreza monetaria para el período comprendido entre el año 2010 y 2015 del nivel nacional, así como para el departamento de Boyacá y la ciudad de Tunja. 
COMPORTAMIENTO DE LA POBREZA MONETARIA

Esta sección describe los resultados de pobreza objetiva monetaria para el período comprendido entre el año 2010 y 2015 del nivel nacional, así como para el departamento de Boyacá y la ciudad de Tunja, con base en la metodología realizada por la Misión para el Empalme de las Series de Empleo (MESEP) presentada en 2011. Estos datos los tiene en cuenta el Gobierno nacional para formular políticas públicas nacionales y territoriales, que permiten focalizar el gasto público con miras a la reducción de la pobreza.
Según el DANE (2016), la LP es el costo per cápita mensual mínimo necesario para adquirir una canasta de bienes alimentarios y no alimentarios que permiten un nivel de vida adecuado. Para 2015, la LP aumentó seis puntos porcentuales, que representó en términos monetarios un incremento de $\$ 11.831$ respecto del año anterior. Para este periodo, la LP nacional fue de $\$ 223.638$. Además, un hogar compuesto por cuatro personas se clasificó como pobre, si su ingreso mensual estaba por debajo de $\$ 985.344$ en la zona urbana y de \$591.008 en la rural (ver Figura 1).

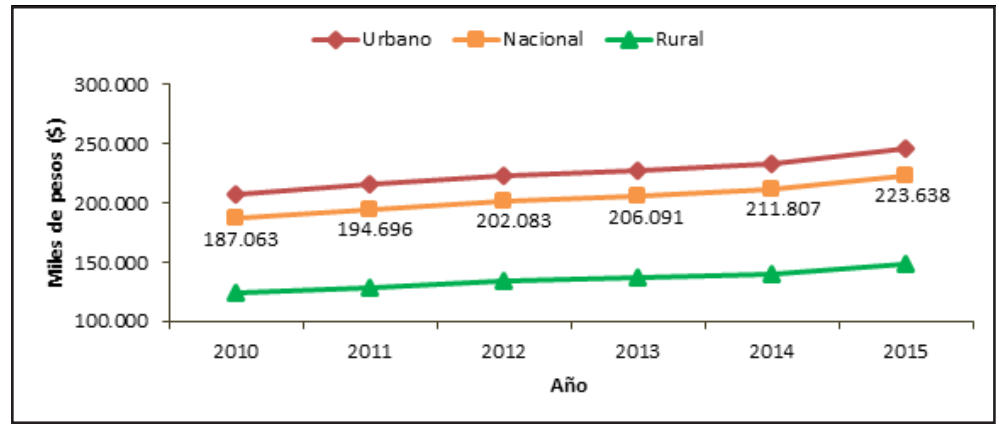

Figura 1. Línea de pobreza: nacional, urbana y rural (2010-2015).

Fuente: DANE-Gran Encuesta Integrada de Hogares.

Cabe señalar que la brecha entre la LP urbana y rural permaneció relativamente constante en el periodo indicado, aunque con una diferencia en el nivel nacional de \$98.584 para 2015. Es decir, las personas de la zona rural pueden acceder a una canasta con una menor cantidad de dinero. Esto se explica por las diferencias en los pre- cios de los bienes entre ambas zonas y, también, por la cantidad de alimentos y demás bienes a los que ambos grupos acceden.

Los valores de la LP se traducen en los porcentajes que el DANE anuncia para los distintos dominios del país. Solo se muestran los resultados en materia de 
pobreza del nivel nacional, urbano, rural, y los del departamento de Boyacá. Según la Figura 2, la pobreza nacional en Colombia se redujo en 9.4 puntos porcentuales del año 2010 al 2015, pasando de $37.2 \%$ a $27.8 \%$. Así mismo, la pobreza disminuyó más en la zona urbana que en la rural.

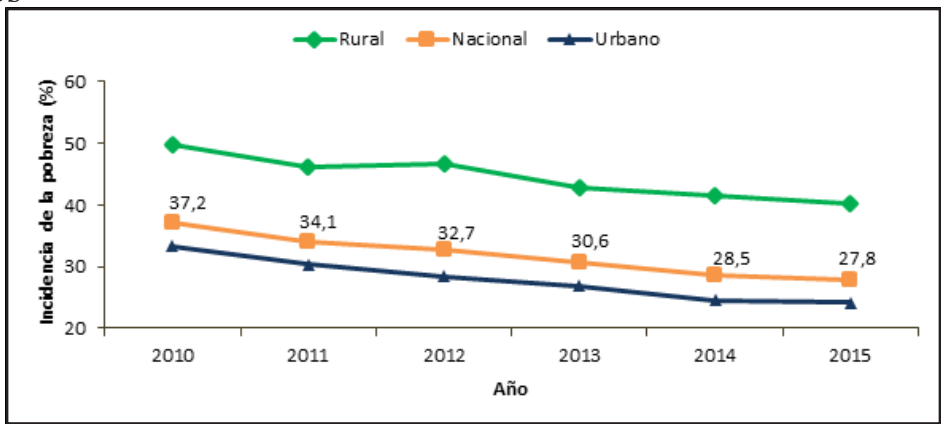

Figura 2. Pobreza: nacional, urbana y rural (2010-2015).

Fuente: DANE-Gran Encuesta Integrada de Hogares.

Ahora, al cotejar los resultados de pobreza del nivel nacional frente a los del departamento de Boyacá, y particularmente de la ciudad de Tunja para el periodo 2010-2015, se puede visualizar en la Figura 3 que la reducción de la pobreza del nivel nacional para el periodo señalado fue de 9.4 puntos porcentuales, ya que pasó de $37.2 \%$ a
$27.8 \%$; mientras que la disminución de las tasas de pobreza en Boyacá fue de 11.7 puntos porcentuales, pasando de $47.1 \%$ a $35.4 \%$; en cambio, para Tunja solo descendió 3.3 puntos porcentuales en el lapso de esos seis años, pasando de $22.1 \%$ en 2010 a $18.8 \%$ en 2015.

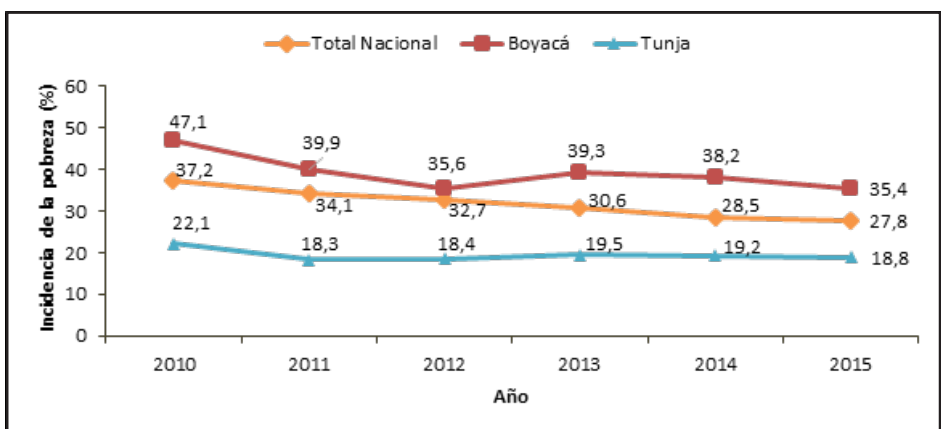

Figura 3. Pobreza en Boyacá, en comparación con el nivel nacional (2010-2015).

Nota: datos expandidos con proyecciones de población elaboradas con base en los resultados del Censo 2005.

Fuente: DANE-Gran Encuesta Integrada de Hogares. 
Por último, cabe indicar que el valor de la LP monetaria para la ciudad de Tunja para el año 2015 se situó en \$233.530. De acuerdo con este valor se obtuvo que el $18.8 \%$ de los tunjanos se situó en condición de pobreza para dicho año. Esto viene a significar que 33.952 habitantes de Tunja son pobres dentro de los 180.594 habitantes que proyectó el DANE con base en los resultados del censo 2005. Es con fundamento en esta información del año 2015 que se confrontan los resultados obtenidos con cada uno de los tres métodos que se comentan en la siguiente sección.

\section{DETALLES METODOLÓGICOS}

En esta sección se explican los detalles metodológicos que se tuvieron en cuenta para calcular la LPS de Tunja para el año 2015. Para ello se subdivide esta sección en dos partes. En la primera se exponen sucintamente los principales aspectos de los tres métodos más utilizados para la obtención de la LPS. En la segunda se tratan los aspectos que se tuvieron en cuenta para la implementación, el diseño y la recolección de información de la encuesta que se utilizó para la estimación de la LPS para Tunja.

\section{Métodos}

Se hizo una revisión bibliográfica relacionada con los métodos más utilizados en el mundo para la obtención de la LPS. Sobre esto, Ravallion y Lokshin $(1999$, p. 2) afirman que es "una paradoja que los economistas asuman por un lado que los individuos son los mejores jueces para apreciar el impacto de las políticas económicas sobre su bienestar y por otro lado se resistan a preguntarles a dichas personas si su bienestar ha mejorado o no".

En ese sentido, al revisar la literatura se ha observado que el análisis de pobreza subjetiva parte de preguntas que se formulan directamente a los individuos sobre la percepción que tienen sobre su nivel de ingreso actual, situación económica y grado de satisfacción respecto de aspectos como salud, empleo o clase social (Ravallion, 1998). Para la agregación de estas percepciones se han utilizado varias preguntas como instrumentos que intentan medir la pobreza subjetiva.

En ese sentido, la LPS es un umbral en términos de ingreso o gasto por debajo del cual las personas son consideradas pobres. Al respecto existen varios métodos ${ }^{3}$ para obtener el ingreso mensual que las personas consideran como mínimo para suplir sus

\footnotetext{
${ }^{3}$ Véanse Prtiz y Marco (2001, pp. 47-53).
} 
requerimientos mínimos. Aquí solo se explican los tres métodos más utilizados en los ejercicios de medición de pobreza subjetiva en el mundo.

\section{Pregunta de ingreso mínimo}

Goedhart, Halberstadt Kapteyn y Van Praag (1977) evalúan la diferencia entre lo que las personas ganan y gastan, y además, interrogan a estas sobre lo que consideran su ingreso mínimo necesario para su familia. La agrupación de los valores proporcionados en la respuesta arroja el ingreso mínimo denotado por que representa el ingreso mínimo subjetivo (IMS) que separa a las personas de ser pobres de no serlo. Estos autores (1977) propusieron la siguiente pregunta: ¿Cuál es el monto de ingreso mínimo (en unidades monetarias) que su familia estima necesario para satisfacer sus necesidades básicas?

La respuesta proporcionada por el entrevistado suele depender del ingreso que percibe el hogar mensualmente. Sin embargo, el entrevistado suele considerar que el ingreso percibido está por debajo del que necesita su familia. Para obtener el valor de la LPS es necesario estimar los parámetros de una ecuación en la que el IMS depende del ingreso corriente que percibe el encuestado y del tamaño de la familia de la que es miembro. Para determinar el valor de la línea se plantea la ecuación 1 :

$$
\ln \left(y_{\min }\right)=\widehat{\beta_{0}}+\widehat{\beta_{1}} \ln y+\widehat{\beta_{2}} \ln f X
$$

Donde, $y_{\min }$ corresponde al ingreso mínimo necesario que el entrevistado considera apropiado para cubrir las necesidades mensuales del hogar; $y$ es el ingreso corriente que percibe el hogar al mes; $\boldsymbol{f}$ es el número de integrantes que conforman el hogar; $\boldsymbol{X}$ corresponde a indicadores socioeconómicos y demográficos de los hogares. De la ecuación 1 se obtiene que el IMS es igual al valor de los ingresos corrientes:

$$
y=y_{\min } \quad \text { [2] }
$$

Según Monge y Ravina (2003), reemplazando los coeficientes estimados de la ecuación 1 en la ecuación 3 se obtiene el $y_{\text {min }}$ o la LPS:

$$
y_{\min }=\exp \left[\frac{\hat{\alpha}+\hat{\pi} \bar{x}}{(1-\hat{\beta})}\right]
$$

Como resultado $y_{\min }$ representa el nivel monetario en el cual los encuestados concebirán sus niveles de ingresos como adecuados. Por ende, este valor será la LPS que indica el valor monetario de inflexión o umbral que permite diferenciar a pobres -las personas que están por debajo de dicho valorde los no pobres. Dicho en otras palabras, las personas que logren un ingreso superior al establecido por la LPS 
conseguirán satisfacer las necesidades básicas y requerimientos mínimos de la familia.

No obstante, este método presenta problemas de sobreestimación. En primer lugar, porque el valor de la respuesta a la pregunta de ingreso mínimo que se obtiene está condicionado al ingreso corriente que el encuestado percibe actualmente y, en consecuencia, tiende a inflarlo en función del ingreso actual (Aguado, Osorio \& Ahumada, 2007). En segundo lugar, no se puede saber con certeza la noción que tiene cada encuestado sobre el "ingreso mínimo". De ahí que Ravallion (1998) advierta que el ingreso no es un concepto bien definido en términos generales. Por lo anterior, el método que se presenta a continuación surge como una solución cualitativa a las restricciones que presenta la formulación de una pregunta cuantitativa del ingreso mínimo.

\section{Pregunta sobre la adecuación del ingreso}

Diseñada por Colasanto, Kapteyn y Van der Gaag (1983), quienes sugie- ren utilizar preguntas cualitativas dirigidas a evaluar categorías de consumo específicas, tales como ingreso, consumo, alimento, vivienda, vestuario, salud, educación. La pregunta y las respuestas propuestas por estos autores las plantearon en los siguientes términos: Según las circunstancias de su hogar, ¿cómo considera su nivel mensual de ingreso/consumo/vivienda... disponible? Y las respuestas: Menos que adecuado, solo adecuado o más que adecuado.

Las líneas son obtenidas a partir de un modelo $\operatorname{logit}^{4}$ que busca la probabilidad de alcanzar un estándar adecuado de vida según los ingresos y/o gastos que tienen los hogares y variables sociodemográficas como el tamaño de la unidad familiar (Grupo de Rio, 2007). Es importante resaltar que la variable al ser dicótoma toma dos valores cuyo valor de 0 y 1 depende de la ausencia o presencia de cierta condición, siendo así $Y=1$ cuando el ingreso actual se considera adecuado o más que adecuado y $Y=0$ cuando el ingreso actual no se considera adecuado. Por ello, se deja $P=0.5$ para establecer que el evento se presente o no. En

\footnotetext{
${ }^{4}$ Los modelos logit o probit se distancian de los modelos de regresión lineal por el método de mínimos cuadrados ordinarios (MCO), debido a que la variable dependiente solo puede asumir dos valores: 0 ó 1. Si se estimara por $\mathrm{MCO}$, calcular la variable dependiente como una variable dicótoma presenta problemas, tales como que el término de error no estaría distribuido normalmente; en segundo lugar, el término de error no tiene varianza constante por lo que se presenta heteroscedasticidad; y por último_la_estimación por MCO no garantiza que el valor de la variable dependiente se encuentre entre 0 y 1 (Enchautegui, 2000). Por ello, se prefiere un modelo Logit y no Probit, porque todas las variables explicativas (Ingreso corriente y tamaño del hogar) son cuantitativas discretas, lo que permite realizar una mejor interpretación de los coeficientes de regresión
} 
consecuencia, siguiendo a Aguado, Osorio y Ahumada (2007) se plantea un modelo cualitativo de variable dependiente discreta binomial logit, tal y como se ve en la ecuación 4 :

$$
Y=\beta_{0}+\beta_{1} X_{1}+\beta_{2} X_{2}
$$

La ecuación 4 se puede sintetizar en $Y=X \beta$. Por tanto, la probabilidad de que $\mathrm{Y}$ sea igual a 1 se encuentra condicionada a los valores de las variables explicativas, tomando la siguiente forma:

$$
\operatorname{Prob}(Y=1)=F(X, \beta)
$$

Y cuando la probabilidad de $\mathrm{Y}$ es igual a cero:

$$
\operatorname{Prob}(Y=0)=1-F(X, \beta)
$$

Donde $F$ denota la función de distribución acumulada. Como en este caso se aplica un modelo logit, la distribución que sigue es:

$$
F(X, \beta)=\frac{e^{X \beta}}{1+e^{X \beta}}
$$

Por ello, la probabilidad de que el evento $\mathbf{Y}=\mathbf{1}$ suceda, se puede expresar de la siguiente manera:

$$
P=\frac{e^{\sum_{k=1}^{k} \beta_{k} X_{k}}}{1+e^{\sum_{k=1}^{k} \beta_{k} X_{k}}}
$$

En cambio, la probabilidad de que el evento $\mathbf{Y}=\mathbf{0}$ ocurra viene dada por la ecuación 7 :

$$
P=\frac{1}{1+e^{\sum_{k=1}^{k} \beta_{k} X_{k}}}
$$

Siguiendo a Enchautegui (2000), la ecuación 7 se expresa como una combinación lineal de $\beta$ (que es el vector de parámetros) y $X$ (es un vector del conjunto de indicadores socioeconómicos y demográficos de los hogares) cuando la probabilidad del evento sea $Y=1$ :

$$
\frac{P}{1-P}=e^{\sum_{k=1}^{k} \beta_{k} X_{k}}
$$

Por último, el ingreso mínimo que los individuos consideran adecuado surge de la combinación lineal exponenciada de los estimadores de coeficientes de regresión y variables explicativas. Haciendo los ajustes correspondientes a la ecuación 7 se tiene:

$$
e^{\ln \left(\frac{P}{1-P}\right)}=e^{\left[\beta_{0}+\beta_{1} X_{1} \bar{x}+\beta_{2} \ln \left(X_{2}\right)\right]}
$$

[9]

Donde, $\beta_{0}$ representa el ingreso mínimo considerado como adecuado que no depende del tamaño del hogar $\left(X_{1}\right)$ ni de su ingreso corriente mensual $\left(X_{2}\right)$. Cabe precisar que representa el promedio de habitantes por hogar, que para el caso de Tunja dio 3.48. Con estas ecuaciones se procedió a estimar la LPS como se muestra en la sección de resultados.

\section{Pregunta de evaluación del ingreso}

Otra opción es la LP de Leyden, que se basa en el siguiente interrogante:

Por favor trate de indicar lo que usted considera una suma apropiada de 
dinero para cada uno de los siguientes casos. Bajo mis (nuestras) condiciones, yo llamaría a un ingreso semanal/mensual/anual (por favor, haga un círculo alrededor del período apropiado), después de impuestos de:

$\begin{array}{ll}\text { Alrededor de } & \text { muy malo } \\ \text { Alrededor de } & \text { malo } \\ \text { Alrededor de } & \text { insuficiente } \\ \text { Alrededor de } & \text { suficiente } \\ \text { Alrededor de } & \text { bueno } \\ \text { Alrededor de } & \text { muy bueno }\end{array}$

Este método supone que cada hogar calcula adecuadamente el ingreso para cada una de las seis situaciones planteadas. Además, supone que los individuos evalúan el ingreso a través de una función de utilidad $\boldsymbol{U}=\boldsymbol{U}(\boldsymbol{y})$ que se puede acotar entre 0 y 1 . La función de utilidad del hogar se puede estimar para cada uno de los ingresos que contestan los hogares. Así, por ejemplo, a la respuesta "muy malo" se le asigna 1/12, a "malo" 3/12, a "insuficiente" 5/12", a "suficiente" 7/12, a "bueno" 9/12; y a "muy bueno" 11/12 (Ortiz \& Marco, 2001).

De esta manera, se tendrán seis puntos en las ordenadas que pertenecen a cada una de las situaciones planteadas, $y$ seis puntos en las abscisas correspondientes a los valores de ingresos asignados por los hogares a cada situación. La forma funcional de la función de utilidad se ajusta a una distribución log-normal que tendrá media $\mu \mathrm{y}$ desviación típica $\sigma$. A la media se le llama parámetro de necesidad del hogar $\mathrm{y}$ será la cifra de ingresos mensuales que conciernen a un nivel de utilidad de 0.5 (Ortiz \& Marco, 2001). En seguida se estıma una recta de regresión que explique el parámetro de necesidad en función del tamaño del hogar y del ingreso del mismo, obteniéndose:

$$
\mu=\widehat{\beta_{0}}+\widehat{\beta_{1}} \log f+\widehat{\beta_{2}} \log y
$$

Donde, $f$ es el tamaño del hogar, $y$ es el ingreso del hogar. Para determinar la LPS se fija un nivel de utilidad que se considere como el mínimo aceptable para el hogar $\boldsymbol{w}^{*}$. Habitualmente, este mínimo se asume con un valor de 0.4 (Martín-Guzmán, Toledo, Bellido, López \& Jano, 1996). Por último, se obtiene que corresponde a la media de todas las desviaciones típicas y se determina el nivel de ingresos $\left(y^{*}\right)$ correspondiente al nivel de utilidad asignado. Por tanto:

$$
\log y^{*}=\mu+\bar{\sigma} v\left(w^{*}\right)
$$

Donde $\boldsymbol{v}\left(\boldsymbol{w}^{*}\right)$ es el valor de una normal estándar que acumula una probabilidad de $\boldsymbol{w}^{*}$. Entonces, reemplazando la ecuación 10 en 11 el $\log y^{*}$ se puede expresar:

$$
\log y^{*}=\widehat{\beta_{0}}+\widehat{\beta_{1}} \log f+\widehat{\beta_{2}} \log y+\bar{\sigma} v\left(w^{*}\right)
$$


Así que la LPS sale de igualar $\log y=\log y^{*}$, que al despejar queda:

$$
\log y=\frac{\beta_{0}+\beta_{1} \log f+\bar{\sigma} v\left(w^{*}\right)}{1-\beta_{2}}
$$

Una de las ventajas de este método es que permite obtener umbrales de pobreza para diferentes tamaños del hogar. Así mismo, por su diseño metodológico, las estimaciones son más robustas, debido a las ponderaciones que hacen de cada una de las situaciones planteadas (Ortiz \& Marco, 2001). De este modo, la LPS se obtiene a raíz de seis valores, que al ponderarse y ajustarse al parámetro de necesidad del hogar y un mínimo aceptable de utilidad para el hogar, es posible que se tenga un valor más ajustado a la realidad, debido a que el encuestado suministra información desde un ingreso "muy malo" a uno "muy bueno".

\section{Diseño y aplicación de la encuesta}

Los datos para determinar la LPS de Tunja para 2015 se obtuvieron a partir de encuestas realizadas a los hogares entre los distintos estratos socioeconómicos de la ciudad. Sin embargo, para estimar el tamaño de la muestra se acudió al Comité de Estratificación de la Alcaldía de Tunja, la cual proporcionó la información de la cantidad de hogares por barrios y estrato en la ciudad. Además, se tuvo en cuenta la información de la población proyectada suministrada por el DANE para
Tunja en 2015, elaborada con base en los resultados del Censo 2005, junto con la información relacionada con el factor de expansión de la Encuesta de Hogares de 2015 para la ciudad de Tunja, para expandir los resultados de las tasas de pobreza.

En este punto, se consideró pertinente tener en cuenta el factor de expansión de la encuesta señalada del DANE, porque se confrontaron las tres LPS obtenidas a partir de los tres métodos con la base de datos de la entidad, para así poder hacer un mejor ejercicio comparativo. Sin embargo, se hizo el factor de expansión propio de la encuesta que se presentó a la población urbana de Tunja. De este modo, se muestran ambos resultados para que el lector pueda tener el panorama de las cifras y así mostrar la consistencia del ejercicio de muestreo realizado. Como se podrá observar en la Tabla 7 , las cifras de pobreza son mayores en la encuesta del DANE, en promedio un $4.97 \%$.

El método de muestreo utilizado fue muestreo simple. Sin embargo, se fraccionó la población por estratos y se ponderó de acuerdo con la cantidad de habitantes residentes en los mismos, con el propósito de que cada estrato tuviera asignado una cantidad de encuestas de forma proporcional al tamaño dentro del total poblacional. Para la construcción del documento se consideró apropiado encuestar a hoga- 
res de todos los estratos, con el fin de ser completamente objetivos, ya que la percepción de pobreza puede ubicarse en cualquier condición y estrato social. De este modo, fueron entrevistadas las personas mayores de edad que integran un hogar (asociándolo como sujeto representativo del mismo). El municipio de Tunja está distribuido en cinco estratos, con un total de 42.748 hogares (ver Tabla 1).

Tabla 1. Cantidad de barrios, hogares y peso de los mismos según estrato.

\begin{tabular}{|c|c|c|c|}
\hline ESTRATO & BARRIOS & HOGARES & $\begin{array}{c}\text { PORCENTAJE DE } \\
\text { HOGARES }\end{array}$ \\
\hline 1 & 19 & 2.878 & $7 \%$ \\
\hline 2 & 28 & 11.838 & $28 \%$ \\
\hline 3 & 66 & 17.233 & $40 \%$ \\
\hline 4 & 33 & 7.559 & $18 \%$ \\
\hline 5 & 11 & 3.240 & $8 \%$ \\
\hline TOTAL & $\mathbf{1 5 7}$ & $\mathbf{4 2 . 7 4 8}$ & $\mathbf{1 0 0} \%$ \\
\hline
\end{tabular}

Fuente: Oficina Asesora de Planeación, Alcaldía de Tunja (2012).

Teniendo en cuenta que la población objetivo fue de 42.748 hogares, se procedió a utilizar el estadístico de prueba zeta $(Z)$, que sigue una distribución de probabilidad normal y cuyos valores se distribuyen formando una campana de Gauss que toma un valor central que coincide con el valor medio de la distribución (Bencardino, 2013). En ese orden de ideas, debido a que la población se conoce, se utilizó la ecuación 14 para obtener el tamaño de la muestra para poblaciones finitas (Morales, 2012).

$$
n=\frac{\left(Z^{2} * p * q\right) * N}{\left(e^{2}(N-1)\right)+Z^{2} * p * q}
$$

Donde,

$Z=1.96$, que representa un nivel de confianza $\alpha=95 \%$,

$e=5 \%$, que significa un error muestral $1-\alpha=5 \%$.

$p=50 \%$. Se asumió que el $50 \%$ de los encuestados están en condición de pobreza.

$q=50 \%$. Se asumió que el $50 \%$ de los encuestados no están en condición de pobreza.

$N=42.748$ hogares. Representa la población. 
Reemplazando los valores en la ecuación 14 , se obtuvo una muestra de $n=380,74$. En total se hicieron 381 encuestas. Respecto de la asignación proporcional, el tamaño de la muestra de cada estrato fue proporcional al tamaño del estrato poblacional que se mostró en la Tabla 1. En conclusión, se aplicaron 26 encuestas a los hogares pertenecientes al estrato uno, 105 al estrato dos, 154 al estrato tres, 67 al cuatro y 29 del cinco, para un total de 381 encuestas aplicadas a la población urbana del municipio de Tunja.
El siguiente paso fue aplicar la encuesta a la población urbana del municipio de Tunja, compuesta por cuatro preguntas. Las cuatro iniciales pretendían garantizar que el perfil del encuestado se ajustara a los criterios establecidos, tales como estrato y mayoría de edad. Las restantes se dirigían a obtener la información necesaria para obtener la LPS, por medio de una pregunta cuantitativa y una cualitativa, bajo los tres métodos comentados en la primera subdivisión de esta sección (ver Figura 4).

\begin{tabular}{|l|l|}
\hline Edad: & $\begin{array}{l}\text { Número de miembros } \\
\text { en el hogar: }\end{array}$ \\
\cline { 1 - 1 } Estrato socioeconómico: $1 \_2{ }_{-}{ }^{3}{ }_{4}{ }_{-} 5_{-}$ & en
\end{tabular}

1. ¿Cuál es el ingreso promedio mensual de su hogar? $\$$

2. El estándar de vida (el nivel mensual de ingreso disponible) para atender las necesidades de su familia es:

R. Menos que adecuado__ Sólo adecuado__ Más que adecuado__

3. ¿Cuál considera usted, en sus circunstancias, es el ingreso mínimo mensual que necesita su hogar para cubrir sus necesidades básicas?

$\$$

4. Por favor trate de indicar lo que usted considera una suma apropiada de dinero para cada uno de los siguientes casos. Bajo sus condiciones, usted llamaría a un ingreso mensual, después de impuestos: ¿Alrededor de cuánto?

Alrededor de Muy malo, Alrededor de Malo

Alrededor de Insuficiente, Alrededor de Suficiente

Alrededor de bueno, Alrededor de Muy bueno

Figura 4. Encuesta aplicada en la cabecera municipal de Tunja.

Fuente: construcción propia con base en Goedhart et al. (1977);

Colasanto, Kapteyn y Van der Gaag (1983); Ortiz y Marco (2001). 
Por último, se pueden citar algunos trabajos interesados en capturar la percepción de la pobreza de los seres humanos, con el fin de tener referentes que nos permitan obtener la LPS. $\mathrm{Al}$ respecto, el Banco Mundial llevó a cabo un estudio mundial, sobre las percepciones de las personas acerca de su nivel de vida y bienestar. El informe se tituló " $\mathrm{La}$ voz de los pobres" y se publicó en 1999. Este recopiló las opiniones y experiencias de más de 40.000 personas pobres en 50 países para el informe sobre el desarrollo mundial 2000-2001. Este estudio marcó un precedente al diferenciarse de otros, debido a que intentó entender la pobreza desde los puntos de vista de los directamente afectados, enviando mensajes para implementar estrategias eficaces de desarrollo social y económico (Banco Mundial, 1999).

En América Latina se han encontrado estudios recientes sobre bienestar subjetivo. En Argentina se construyó un índice de bienestar económico que mide el nivel de satisfacción de la población respecto de unos aspectos (ingresos, educación, trabajo, entre otros) de acuerdo con el nivel de importancia que la propia población le brinda a los mismos. El valor del IBE a diciembre de 2005 se ubicó en 45 puntos sobre una escala de 0 a 100 Giarrizo, 2007)

En México se hizo un trabajo basado en una encuesta aplicada a 1540 personas pertenecientes a cinco estados del centro y del sur, buscando analizar la evaluación que cada persona hace de su condición de vida, que no solo se basa en su ingreso actual, sino en su situación histórica, grupo de referencia y aspiraciones materiales futuras. Al respecto, Rojas y Jiménez (2008) encontraron que la pobreza subjetiva no coincide con los tres criterios determinados por la Secretaría de Desarrollo Social, que mide la pobreza de acuerdo con tres criterios de pobreza de imputación/presunción, destacándose que la pobreza subjetiva es mayor frente a la reportada por la Secretaría de ese país.

Para Colombia, Aguado, Osorio y Ahumada (2007) propusieron un diseño preliminar de una encuesta para analizar la pobreza desde un enfoque subjetivo, que puede ser replicada en Colombia y América Latina. Pinzón (2011) estableció los determinantes de la pobreza subjetiva desde el enfoque de capacidades y la economía de la felicidad a partir de la Encuesta Nacional de Calidad de Vida del DANE en 2011, en la que se menciona que el $80 \%$ de los hogares colombianos que no se consideran pobres bajo la línea de pobreza monetaria, se siente pobre, por lo que permitió encontrar que aunque el ingreso tiene una mayor importancia en la percepción de pobreza de los colombianos, estar bien nutrido se constituye como la capacidad elemental que deben te- 
ner las personas para desarrollar otras capacidades y, además, que en las regiones con alta presencia de violencia, el desplazamiento forzoso incide en la percepción de pobreza.

Por último, González (2013) llevó a cabo un estudio en el cual se estimó una LPS a través de la encuesta de percepción ciudadana para Cali de 2008, que arrojó que un hogar era pobre si sus ingresos son menores a $\$ 872.545$ pesos. Además, encontró una relación negativa entre el tamaño del hogar y el ingreso que perciben los hogares. Como puede observarse, los estudios relacionados con la pobreza subjetiva no son abundantes en el país y en el caso particular no existe uno para el municipio de Tunja, de allí la pertinencia de este documento, pues abarca una dimensión de la pobreza que no es fácilmente observable.

\section{ANÁLISIS DE RESULTADOS}

Al aplicar la encuesta se supuso que las personas encuestadas entendieron las preguntas y tuvieron la capacidad para comprender y evaluar su propia condición $\mathrm{y}$, además, respondieron de forma veraz a cada una de las preguntas, pues no tenían incentivos para mentir. Fuera de las respuestas de los encuestados, se detectaron elementos interesantes, que se muestran en la Tabla 2. Por ejemplo, se puede observar que el ingreso mensual medio reportado por los hogares del estrato uno fue de $\$ 538.848$, mientras que para el estrato cinco fue de $\$ 5^{\prime} 572.414$, lo que indica una brecha de $934.1 \%$; así mismo, se puede notar que el ingreso mensual del estrato uno está por debajo del promedio de la ciudad en 293 $\%$. Esto revela las brechas en materia de ingresos de un estrato a otro.

Tabla 2. Ingreso mensual y tamaño del hogar promedio por estratos en Tunja (2015).

\begin{tabular}{|c|c|c|c|c|c|c|}
\hline Variable & Estrato1 & Estrato 2 & Estrato 3 & Estrato 4 & Estrato 5 & $\begin{array}{c}\text { Promedio } \\
\text { Tunja }\end{array}$ \\
\hline $\begin{array}{c}\text { Ingreso } \\
\text { mensual }\end{array}$ & $\$ 538,848$ & $\$ 1,165,171$ & $\$ 1,872,948$ & $\$ 3,456,716$ & $\$ 5,572,414$ & $\$ 2,117,936$ \\
\hline $\begin{array}{c}\text { Tamaño } \\
\text { hogar }\end{array}$ & 4.27 & 3.69 & 3.28 & 3.22 & 3.48 & 3.48 \\
\hline
\end{tabular}

Fuente: elaboración propia con los datos obtenidos de la encuesta aplicada.

Así mismo, en la Tabla 2 se ve el número promedio de miembros del hogar por estratos, y se nota que entre más bajo sea el estrato, más miembros hay. El estrato uno tiene 4.27 miembros, mientras que el estrato cinco tiene 
3.48, lo que significa que la diferencia es de $18.5 \%$. Esto indica que el menor ingreso percibido por parte de los hogares de estrato uno lo tienen que repartir entre más miembros; en contraste con lo que sucede en el estrato cinco.

\section{Estimación de la LPS por el mé- todo del ingreso mínimo}

Este método busca evaluar la diferencia entre lo que las personas ganan y gastan versus lo que ellos consideran un ingreso mínimo absoluto para su familia. A partir de las respuestas proporcionadas por los habitantes de Tunja a la pregunta número 2 de la Figura 4, se estimó un modelo econométrico por mínimos cuadrados ordinarios, que permitió estimar los parámetros de la ecuación 1, para reemplazar sus valores en las ecuaciones 2 y 3 .

Antes de mostrar los resultados se debe advertir que el IMS depende del ingreso corriente que el encuestado percibe, el tamaño de la familia de la que es miembro y un indicador socioeconómico y demográfico de los hogares que en este caso es el tamaño promedio de los hogares en Tunja (3.48). Es de notar que existe una relación positiva y cóncava entre la función de ingreso actual y los valores mínimos de ingresos proporcionados. En la Tabla 3 se muestran los estimadores obtenidos para este método.

Tabla 3. Regresión lineal sobre la pregunta de ingreso mínimo.

\begin{tabular}{|c|c|c|c|}
\hline \multicolumn{4}{|c|}{ Dependent Variable: Log(Ymin) } \\
Observations & 381 \\
\hline Variable & Coefficient & Std. Error & t-Statistic \\
\hline Constant & $4.643^{\star * *}$ & -0.301 & -15.4252492 \\
\hline $\operatorname{Ln}(\mathrm{Y})$ & $0.676^{\star * *}$ & -0.0205 & -32.9756098 \\
\hline $\operatorname{Ln}(\mathrm{f})$ & $0.0802^{* * *}$ & -0.0107 & -7.4953271 \\
\hline R-squared & 0.745 \\
\multicolumn{4}{|c}{$\mathrm{***} p<0.01,{ }^{* *} \mathrm{p}<0.05,{ }^{*} \mathrm{p}<0.1$}
\end{tabular}

Fuente: cálculos propios en stata 12 .

Los estimadores son estadísticamente significativos. Por lo tanto, se procedió a reemplazarlos dentro de las ecuaciones señaladas para obtener el valor de la LPS. De este modo, se obtuvo que la LPS fue de \$3.938.052 que, dividida entre cuatro miembros, atendiendo a la definición de hogar del DANE, se obtuvo una LP per cápita de $\$ 984.513$. 
Estimación de la LPS por el método de adecuación del ingreso

Este método busca ser una alternativa de solución a las restricciones que presenta el método del ingreso mínimo, por medio de la formulación de una pregunta sujeta a una respuesta cualitativa que evalúa la percepción que tienen las personas sobre su ingreso actual. A partir de las respuestas proporcionadas por los habitantes de Tunja a la pregunta 3 de la Figura 4 , se estimó un modelo econométrico binomial, a pesar de que existen tres opciones de respuestas, ya que no se encontró una diferencia significativa entre el modelo binomial frente al multinomial. Se decidió que si los encuestados contestaban que su ingreso es adecuado o más que adecuado, to- mara valores de 1 ; mientras que para la opción menos que adecuado, de 0 . Así, la probabilidad será cuando el evento se presente o no.

Además, cuando la variable dependiente es dicótoma, el valor esperado de la variable de acuerdo con las variables explicativas no es lineal en parámetros, por lo que se usó el método de máxima verosimilitud, que busca que los valores de los estimadores maximicen la probabilidad de que el valor observado de la variable dependiente sea predicho según los valores de las variables independientes (Enchautegui, 2000). La estimación del Logit para el método de adecuación del ingreso arrojó los resultados que se muestran en la Tabla 4.

Tabla 4. Estimación del Logit del método de adecuación del ingreso.

\begin{tabular}{|c|c|c|c|}
\hline \multicolumn{4}{|c|}{ Dependent Variable: LOGIT_Tunja Dummy } \\
\hline Observations & 381 & & \\
\hline Variable & Coefficient & Std. Error & t-Statistic \\
\hline Constant & $-28.04^{\star \star \star}$ & -3.399 & 8.249485143 \\
\hline $\operatorname{Ln}(Y)$ & $2.118^{* * *}$ & -0.244 & -8.68032787 \\
\hline Tamaño hogar & $-0.576 * \star \star$ & -0.111 & 5.189189189 \\
\hline
\end{tabular}

Fuente: cálculos propios en stata 12.

Según Aguado, Osorio y Ahumada (2007), se puede estimar la LPS para el modelo de adecuación del ingreso a partir de las ecuaciones 4 a 9. Con los resultados obtenidos se obtuvo la LPS por este método, dando como resultado una LPS para el hogar de \$2’313.481 y una LPS per cápita de $\$ 578.310$. Por 
medio de los valores se obtiene la significancia individual de los coeficientes del modelo, que fueron mayores a 2 , en términos absolutos, lo que arroja un nivel de confianza del $95 \%$.

\section{Estimación de la LPS por el método de evaluación del ingreso}

La ventaja que ofrece este método es que se pueden obtener líneas de acuerdo con el tamaño del hogar. Con las respuestas de la pregunta 4 de la Figura 4 , se procedió a aplicar el procedimiento señalado para reemplazar los valores en las ecuaciones 10 a 13 . Igualmente, el valor que se asumió para fue 0.4. La estimación del $p a$ rámetro de necesidad del hogar fue de $\mu=0.5421$ y la media de todas las desviaciones típicas fue de $\bar{\sigma}=0.2708$. Al hacer la regresión por $\mathrm{MCO}$ de la ecuación 12 se obtuvieron los resultados indicados en la Tabla 5 .

Tabla 5. Regresión lineal sobre la pregunta de evaluación del ingreso

\begin{tabular}{|c|c|c|c|}
\hline \multicolumn{4}{|c|}{ Dependent Variable: Ln(Ymin) } \\
Observations & 381 \\
\hline Variable & Coefficient & Std. Error & t-Statistic \\
\hline Constant & $8.4540^{* * *}$ & 0.3566 & 23.70254 \\
Ln(Y) & $0.4013^{* * \star}$ & 0.0243 & 16.47037 \\
\hline Ln(f) & 0.0511 & 0.0406 & 1.25932 \\
\hline R-squared & 0.4136 \\
\multicolumn{4}{|c}{$* * 0.01,{ }^{* *} p<0.05,{ }^{*} p<0.1$}
\end{tabular}

Fuente: cálculos propios en stata 12.

Con estos valores se obtuvo la LPS por este método, teniendo en cuenta el número de miembros del hogar. De este modo, en la Tabla 6 se presentan cuatro columnas. En la primera se muestra el valor de la LPS estimada para cada uno de los distintos tamaños del hogar (columna dos). En la tercera columna se muestra el porcentaje del número de integrantes que conforman el hogar, que va de uno a diez (al respecto se encontró que en la ciudad el $34.2 \%$ de los hogares tunjanos tiene tres miembros, seguida de los hogares conformados por cuatro miembros). Y en la cuarta columna se detalla el valor 
Apuntes CENES Volumen 36, Número 64

julio - diciembre 2017. Págs. 253-282

de la LPS per cápita para cada uno de los tamaños del hogar.

Tabla 6. Valores de la LPS para distintos tamaños de hogar

\begin{tabular}{|c|c|c|c|}
\hline LPS & TH & $\%$ & LPS per cápita \\
\hline$\$ 1,626,217$ & 1 & $8.2 \%$ & $\$ 1,626,216.9$ \\
\hline$\$ 1,725,485$ & 2 & $12.1 \%$ & $\$ 862,742.45$ \\
\hline$\$ 1,786,339$ & 3 & $34.2 \%$ & $\$ 595,446.25$ \\
\hline$\$ 1,830,812$ & 4 & $\mathbf{2 4 . 7 \%}$ & $\$ \mathbf{4 5 7 , 7 0 3 . 1 0}$ \\
\hline$\$ 1,866,070$ & 5 & $15.2 \%$ & $\$ 373,213.99$ \\
\hline$\$ 1,895,381$ & 6 & $3.1 \%$ & $\$ 315,896.82$ \\
\hline$\$ 1,920,522$ & 7 & $1.0 \%$ & $\$ 274,360.27$ \\
\hline$\$ 1,942,569$ & 8 & $0.3 \%$ & $\$ 242,821.17$ \\
\hline$\$ 1,962,227$ & 9 & $0.5 \%$ & $\$ 218,025.18$ \\
\hline$\$ 1,979,979$ & 10 & $0.5 \%$ & $\$ 197,997.91$ \\
\hline \multicolumn{3}{|c|}{ Promedio LPS } \\
\hline
\end{tabular}

Fuente: cálculos propios.

No obstante, es preciso destacar el valor de la LPS per cápita de aquellos hogares que tienen cuatro miembros, por el hecho de que el DANE establece este criterio para obtener la LP del hogar. De modo que si se quiere hacer un contraste, se deberá tomar el valor de $\$ 457,703$ como la LPS por este método.

\section{Contraste entre la LPS y LP mo- netaria en Tunja}

Finalmente, se consideró pertinente contrastar el número de tunjanos que se encuentran en situación de pobreza bajo la LP y los distintos métodos uti- lizados en el documento para la obtención de la LPS. Al respecto, se tomaron como referencia los siguientes valores: i) LP \$233.530 que corresponde a la LP urbana per cápita estimada por el DANE para el dominio urbano de Tunja en 2015; ii) LPS por el método del ingreso mínimo $\$ 984,513$; iii) LPS por el método de adecuación del ingreso $\$ 578,310$; iv) LPS por el método de evaluación del ingreso $\$ 457,703$.

Como se señaló en la sección cuatro, se tuvo en cuenta la información relacionada con el factor de expansión de la Encuesta de Hogares de 2015 para la ciudad de Tunja, para expandir los 
resultados de las tasas de pobreza. Así mismo, el documento hizo el factor de expansión propio de la encuesta realizada. Con los valores de las líneas obtenidas con los tres métodos, se procedió a contrastarlos frente al porcentaje de pobres conseguido por el DANE para Tunja en 2015 (18,79\%). En la Tabla 7 se presenta un resumen de los valores hallados de las líneas, así como el porcentaje de pobres.

Tabla 7. Valor líneas y porcentaje de pobres para la ciudad de Tunja 2015.

\begin{tabular}{|c|c|c|c|c|}
\hline Ítem & DANE & Método 1 & Método 2 & Método 3 \\
\hline Valor línea de pobreza & $\$ 221,950$ & $\$ 982,099$ & $\$ 578,370$ & $\$ 457,703$ \\
\hline $\begin{array}{c}\text { Porcentaje pobres con factor de } \\
\text { expansión DANE }\end{array}$ & $18.79 \%$ & $80.53 \%$ & $59.60 \%$ & $48.77 \%$ \\
\hline $\begin{array}{c}\text { Porcentaje pobres con factor de } \\
\text { expansión propio }\end{array}$ & No aplica & $76.37 \%$ & $53.54 \%$ & $44.09 \%$ \\
\hline
\end{tabular}

Fuente: cálculos propios utilizando la Base de Datos de Hogares de 2015-DANE.

En síntesis, con los resultados obtenidos se observa que por el método del ingreso mínimo el porcentaje de pobres, utilizando el factor de expansión de la encuesta de hogares de 2015 del DANE, sería del $80.53 \%$; mientras que con el factor de expansión del documento fue de $76.37 \%$. De todos modos, con esta línea habría un $61.74 \%$ y $57.58 \%$ más de pobres, recurriendo al factor de expansión del DANE $\mathrm{y}$ al del documento respectivamente. A partir de la estimación de la LPS por el método de adecuación del ingreso se obtuvo, con el factor de expansión del DANE, un $59.6 \%$ de pobres en Tunja; en cambio con el factor de expansión del documento habría un $6.06 \%$ me- nos de pobres, es decir $53.54 \%$. Con estos resultados, contrastándolos con el dato del DANE para 2015 (18.79\%), habría un $40.81 \%$ y $34.75 \%$ más de pobres respectivamente. Respecto de la estimación de la LPS por el método de evaluación del ingreso, se halló un porcentaje de pobres en Tunja de $48.77 \%$, usando el factor de expansión del DANE, y con el factor de expansión del documento un $44.09 \%$.

Es preciso resaltar que en el caso del primer método, la LPS estimada suele estar por encima, por lo que su resultado se desprende de lo que entiende el entrevistado por "ingreso mínimo". En el caso del segundo método, su re- 
sultado se ajusta a una probabilidad del 0.5 de ser o no pobre. En cuanto al tercer método, la LPS surge de acotar los resultados entregados por los entrevistados a un nivel dado de utilidad, lo cual permite registrar seis datos de ingresos por parte del encuestado y, de este modo, hacer un ejercicio un tanto más robusto para obtener una LPS a partir de la ponderación de cada uno de los resultados suministrados.

Sin embargo, no se dice que ningún método es preferible a otro. Nótese que hay arbitrariedad en la selección del método y consideraciones "normativas", es decir, el diseñador del instrumento de medición de la pobreza asume un juicio de valor, porque él lo considera adecuado para describir la realidad. No obstante, lo que sí se presume al haber encuestado a los tunjanos es que los resultados arrojados por los tres métodos revelan que la percepción sobre lo que debería ser el ingreso mínimo es superior al que considera el DANE, lo que vendría a significar que hay más personas que requieren asistencia pública para mitigar las consecuencias negativas de la pobreza y, por ende, el gobierno de Tunja no debería contentarse con las cifras del DANE, sino complementar su medición con la LPS y así lograr identificar a aquellos que no clasificaron dentro del $18.79 \%$ estimado por el DANE, para proponer políticas públicas que permitan mejorar el bienestar de los excluidos de las cifras oficiales.

\section{CONCLUSIÓN}

En este documento se estimó la LPS urbana de Tunja para el 2015 a partir de tres métodos, con el fin de contrastar los resultados oficiales suministrados por el DANE frente a los surgidos de la percepción de los tunjanos. Los resultados obtenidos evidencian que, por lo menos, el gobierno de Tunja no debería ni conformarse ni contentarse con los descensos de las tasas de pobreza que viene anunciando el DANE.

Lo anterior debido a que de la información que arroja el indicador del porcentaje de pobreza les sirve a los gobiernos para cuantificar el número de personas que requieren asistencia y seguridad social subsidiada a través de la política pública. De ahí la relevancia de los hallazgos del documento que agrega a la percepción de inconformismo y escepticismo que se sentía en la sociedad tunjana sobre las cifras oficiales de pobreza, un dato que ameritaría cuestionarse sobre los aspectos metodológicos que sustentan los resultados de la pobreza presentados por el DANE, puesto que, al parecer, no estarían teniendo en cuenta un número importante de personas que necesitan ayuda por parte del Estado.

Cuantificando el porcentaje de pobres en Tunja que requieren dicha asistencia, a partir de los tres métodos planteados en este documento se estimaron unas brechas significativas entre los 
cálculos del DANE y los del documento. De todos modos, en el caso en el que se minimizan las brechas, para el método de evaluación del ingreso, se encontraría un $48.77 \%$ al aplicar el factor de expansión del DANE o un $44.09 \%$ con el factor del documento. Así que de tomarse las estimaciones del documento como ciertas, se estaría desconociendo alrededor de un $29.9 \%$ o $25.3 \%$, que vendría a representar a 54142 ó 45690 personas que deberían considerarse como pobres, ya que su ingreso corriente mensual es inferior al ingreso mínimo que suponen como necesario para suplir sus gastos mínimos.
Finalmente, el desarrollo de este documento puede considerarse como un primer acercamiento al análisis de la percepción subjetiva de la pobreza por parte de los individuos. Sin embargo, debido a limitaciones presupuestarias, en el documento no se pudo considerar la percepción de la pobreza para el sector rural, indispensable para establecer un mejor mapa de las necesidades de la población tunjana. De ahí que se espere que el gobierno de la ciudad de Tunja encuentre en este estudio un referente para formular políticas dirigidas a mejorar el bienestar de quienes más lo necesitan. 
Apuntes CENES Volumen 36, Número 64

julio - diciembre 2017. Págs. 253-282

\section{REFERENCIAS}

Aguado, L., Osorio, A.M. \& Ahumada, J. (2007). Medición de pobreza a partir de la percepción de los individuos: Colombia y el Valle del Cauca de la línea de pobreza subjetiva. Economical Analysis Working Papers, 11, 1-22.

Alcaldía de Tunja. (2012). Cantidad de barrios, hogares y peso de los mismos según estrato. Tunja, Colombia: Oficina Asesora de Planeación.

Banco Mundial. (1999). La voz de los pobres, ¿Hay alguien que nos escuche? España: Mundi-Prensa.

Bencardino, C. M. (2013). Estadística y muestreo. Bogotá: ECOE.

Casas, J. \& Barichello, R. (2015, ene-jun.). Hacia una noción sobre la pobreza. Apuntes del Cenes, 34(59), 39-62.https://doi.org/10.19053/22565779.2784

Colasanto, D., Kapteyn, A. J. \& Van der Gaag, J. (1983). Two Subjective Definitions of Poverty: Results from the Wisconsin Basis needs Study. Ter Discussie FEW, 83(18).

DANE. (2015). Microdatos anonimizados de Encuestas de Hogares. Recuperado de http://190.25.231.249/pad/index.php/catalog/161.

DANE. (2016). Boletín técnico de pobreza monetaria. Bogotá: DANE.

Enchautegui, M. (2000). Módulo de estudio sobre modelos Probit y Logit. Puerto Rico: Universidad de Puerto Rico.

Feres, J. \& Mancero, X. (2001a). Enfoques para la medición de la pobreza, breve revisión de la literatura. Comisión Económica para América Latina y el Caribe, Serie de Estudios Estadisticos y Prospectivos, 4(18).

García, M. (2002). El bienestar subjetivo. Escritos de Psicología, (6), 18-39.

Giarrizo, V. (2007). La pobreza subjetiva en Argentina: una aproximación al bienestar de la población. En XI Jornadas de Epistemología de la Economía. FCE/UBA.

Goedhart, T., Halberstadt, V., Kapteyn, A. \& Van Praag, B (1977). The poverty line: concept and measurement. The Journal of Human Resources, 12, 503-520 https://doi. org/10.2307/145372

González, O. A. (2013). Un acercamiento a la pobreza subjetiva en Cali 2008: ¿Cali cómo vamos en pobreza? Trabajo de grado. Universidad del Valle. Recuperado de http://hdl.handle.net/10893/5885 
Grupo de Río. (2007). Compendio de mejores prácticas en la medición de la pobreza. Recuperado de http://dds.cepal.org/infancia/guia-para-estimar-la-pobreza-infantil/ bibliografia/capitulo-II/Grupo $\% 20 \mathrm{de} \% 20$ Expertos $\% 20$ sobre $\% 20$ Estadisticas $\% 20$ de $\% 20$ Pobreza $\% 20$ (Grupo\%20de\%20Rio)\%20(2007)\%20Compendio\%20 de $\% 20$ mejores $\% 20$ practicas $\% 20$ en $\% 201 \mathrm{a} \% 20$ medicion $\% 20 \mathrm{de} \% 201 \mathrm{la} \% 20$ pobreza.pdf

Instituto Nacional de Estadística de Uruguay -INE- (s.f). Determinación de la línea de pobreza. Programa para el Mejoramiento de los Sistemas de Información Socioeconómica en el Uruguay. CEPAL/INE/BID. Recuperado de http://www.ine.gub. uy/biblioteca/metodologias/taller\%20pobreza/pobreza\%20capitulo2.pdf

Martín-Guzmán, P., Toledo, M. I., Bellido, N., López, F. J. \& Jano, D. (1996). Desigualdad y pobreza en España. Estudio basado en las Encuestas de Presupuestos Familiares de 1973-74, 1980-81 y 1990-91. España: INE y Universidad Autónoma de Madrid.

Monge, A. \& Ravina, R. (2003). Más allá del componente objetivo en la medición de la pobreza: análisis geográfico de las dimensiones objetiva y subjetiva de la pobreza en el Perú. Departamento de Economía, Universidad del Pacífico. Chapters of Books, 1, 61-100.

Morales, P. (2012). Tamaño necesario de la muestra: ¿Cuántos sujetos necesitamos? Estadística aplicada a las ciencias sociales. Madrid: Universidad Pontificia Comillas.

Ortiz, S. \& Marco, R. (2001). La medición estadística de la pobreza. Madrid: Visión Net.

Pinzón, L. F. (2011). Los determinantes de la pobreza subjetiva en Colombia: un estudio desde el enfoque de las capacidades y la economía de la felicidad. Recuperado de http://bibliotecadigital.univalle.edu.co/bitstream/10893/7081/1/0461969-p.pdf

Ravallion, M. (1998). Poverty Lines in Theory and Practice. World Bank Living Standards Measurements. Study Working Paper 133. Washington D.C.: World Bank. https://doi.org/10.1596/0-8213-4226-6

Ravallion, M. \& Lokshin, M. (1999). Identifying Welfare Effects from Subjective Questions. World Bank Policy Research Papers Series 2301. Washington D.C.: World Bank.https://doi.org/10.1596/1813-9450-2301

Rojas, M. \& Jiménez, E. (2008, jul.-dic.). Pobreza subjetiva en México: el papel de las normas de evaluación de ingreso. Perfiles Latinoamericanos, (32), 11-33. 